\title{
A Novel Taxonomy and Comparison Method for Ranking Cloud Computing Software Products
}

\author{
Ehsan Arianyan, Mohammad Reza Ahmadi, and Davood Maleki \\ Iran Telecommunication Research Center (ITRC) \\ \{ehsan_arianyan,mahmadi,dmaleki\}@itrc.ac.ir
}

\begin{abstract}
Cloud computing as a new and modern technology has become a focus of attention and a platform for future services. Cloud computing services have been categorized into three main groups of services, which are infrastructure, platform, and software. Each service has its own unique characteristic while all services have also some common characteristics and challenges. Such characteristics create a complicated combination of features to serve selection of cloud solutions. This paper presents a new feature based taxonomy which facilitates the process of cloud software product selection. More precisely, Hierarchical Cloud Taxonomy Engine is the proposed method to help developers and consumers choose their appropriate product according to their needs and based on real capabilities of different cloud computing products. Moreover, this paper proposes a new evaluation mechanism which sorts the cloud products according to the customer's needs and requirements. Besides, this paper presents a classification model for cloud features and subsequently presents a comparison between some business and research cloud products as a case study. Results of comparisons show that our proposed techniques facilitate the process of cloud product selection.
\end{abstract}

Keywords: Cloud Computing, Comparison, Taxonomy, Service.

\section{Introduction}

Cloud computing is becoming a powerful network architecture to perform large-scale and complex computing [1]. Cloud computing is a model for enabling ubiquitous, convenient, on-demand network access to a shared pool of configurable computing resources (e.g., networks, servers, storage, applications, and services) that can be rapidly provisioned and released with minimal management effort or service provider interaction. Cloud computing brings a number of advantages to consumers in terms of accessibility and elasticity [2]. Cloud Computing is the result of evolution and adoption of existing technologies and paradigms. The goal of cloud computing is to allow users to take advantage from all of these technologies without the need for deep knowledge about or expertise with each one of them. The cloud aims to cut costs, and help the users focus on their core business instead of being impeded by IT obstacles [3, 4]. Cloud offers many strong points: infrastructure flexibility, faster deployment of applications and data, cost control, adaptation of cloud resources to real needs, improved productivity, etc. In general, cloud model is composed of five essential characteristics: On-demand selfservice, Broad network access, Resource pooling, Rapid elasticity, Measured service, three service models: Software as a Service (SaaS), Platform as a Service (PaaS), Infrastructure as a Service (IaaS) and four deployment models: Private cloud, Community cloud, Public cloud and Hybrid cloud[5-7].

Many big players of computer industry such as Google, Microsoft, Amazon, VMware, and Intel are joining the development of cloud services [8]. Google Apps offer web-based applications and products for techniques related to cloud computing, such as management, migration, and etc. [9]. However, due to the existence of various cloud computing 
products and services, comparing them and finding the best one is hard [10]. Often developers face obstacles such as compatibility issues to find the best product for their services which may hinder them from concentrating on their own service development tasks. Hence, developing a service characteristic evaluation system is an increasingly important area in cloud computing field. In this direction, preparing a taxonomy service based on characteristics of cloud computing products is a challenging task. Taxonomy is the science of categorization, or classification of things based on a predefined system. Typically, taxonomy contains a controlled vocabulary with a hierarchical tree-like structure [11].

In this paper, we examine the available cloud computing technologies and present a taxonomy associated with the main characteristics of them. Moreover, a new multi-level and hierarchical circular based taxonomy is presented to describe the key concepts about cloud computing, which helps to easily and quickly compare cloud computing products. We present a new taxonomy that its characteristics are extracted from a thorough research of conventional technologies for creating cloud computing infrastructures such as VMware, Microsoft private cloud, and Openstack. This taxonomy can be used to survey and compare several existing cloud computing products and services developed by various projects world-wide such as NIST, Google, and Amazon. First level of our taxonomy is related to the main characteristics of cloud computing software products. These features include: deployment model, technical features, security features, service features, management features, financial features, backup \& recovery model, and legal aspects as well as guarantee \& support. In the second and third levels, each feature of previous level has been expanded with more details. Rest of the paper is organized as follows: In Section 2.0, we present related works. Section 3.0 describes our proposed taxonomy Model: HCTE (Hierarchical Circular Taxonomy Engine). Section 4.0 presents the elements of HCTE model including the major characteristics of cloud computing products. Section 5.0 presents the proposed cloud product comparison process. Section 6.0 explains our proposed mechanism to evaluate cloud products using HCTE. Final section concludes and recommends future works.

\section{Related Work}

Liu et al. have developed taxonomy of NIST in reference architecture for cloud computing as an Actor/Role based model [11]. They have proposed taxonomy via hierarchical tree-like structure. In the NIST cloud computing reference architecture, a four-level taxonomy is presented to describe the key concepts about cloud computing. Level 1 is role of cloud actors, which is consisted of five major participating actors: cloud consumer, cloud provider, cloud broker, cloud auditor and cloud carrier. Level 2 is activity of the cloud actors which includes service deployment, service orchestration, cloud service management, security and privacy. Level 3 is component, which refer to the specific processes, actions, or tasks that must be performed to meet the objective of a specific activity. Level 4 is sub-component, which presents a modular part of a component. However, this taxonomy does not provide a comprehensive category that applies all features of cloud computing products. Also it does not help developers and consumers to choose their appropriate product or service according to their needs and based on real capabilities of different cloud computing tools and services. Authors in [12] have proposed Intel's cloud computing taxonomy that classifies the breadth of existing cloud technologies for Intel's IT environment and for only Intel's products and services. Authors in $[8,13]$ have explored a taxonomy and comparison for cloud computing services via a tree-structured taxonomy. They have organized characteristics of cloud computing services and proposed a tree-structured taxonomy. This work relies on quick classifications, easier compare with more detailed characteristics and hierarchies, but at the deeper levels of the tree, the difference between the characteristics become more 
blurred. Also, it does not provide a comprehensive category that applies all features of cloud computing products. Authors in [14] have executed a perspective study on cloud computing and proposed an ontology for it. In their model each of the cloud providers have their own set of pricing, billing, flexibility, support, and other important characteristics for their service model. However, these features do not provide a comprehensive list of characteristics for cloud computing products. Authors in [15] have proposed a table-based taxonomy for choosing a suitable cloud provider. Also, the comparison of significant features has offered by them. However, this taxonomy does not provide a comprehensive category that applies all features of cloud computing products. Authors in [16] have offered various table-based classifications for cloud computing and have provided a technical comparison between different infrastructures technologies, solution providers, SaaS \& PaaS providers, and different open source clouds. There are nine evaluation features in their taxonomy incluing: computing architecture, virtualization management, service, load balancing, fault tolerance, interoperability, storage, security, and programming framework. This survey only provides information to evaluate the cloud systems. Authors in [17] have provided discussions about cloud products and have explained their dimensions for comparison. They have also analyzed some listed products. Fifteen dimensions used in this paper for comparison are: resource offered, level of virtualization, flexibility, ease of solution, legacy support, scaling, integration, standards, lock-in, interoperability, SLAs, redundancy, security, resource billing, and other issues such as software. This analysis explains various differences between cloud products and does not focus on component technologies in a vacuum. They only emphasis on theoretical part of classification and does not propose any tool or solution for providers and users. However, this paper provides a comprehensive classification of cloud characteristics and proposes a new taxonomy that considers all features of cloud computing products and services including deployment model, technical features, security features, service features, management features, financial features, and legal aspects as well as guarantee $\&$ support. Our proposed taxonomy provides a comprehensive solution that easily and quickly compares any number of cloud computing products. Moreover, it helps cloud developers and users to select their appropriate product according to their needs and based on real capabilities of different cloud software tools. In addition, the proposed taxonomy offers common terminologies for different cloud products, which eases the process of classification. Also, it is easily extendable and can be used for evaluation in other cloud domains such as cloud services.

\section{Proposed Taxonomy Model: $\mathrm{HCTE}^{1}$}

In this section, we introduce our proposed taxonomy model which we call it Hierarchical Cloud Taxonomy Engine (HCTE). HCTE is a useful tool for the process of cloud product comparison and can be used for classifying various cloud solutions from different aspects. In HCTE model, the characteristics of cloud products are classified into eight major groups. Each of these major characteristics groups are consisted of some different specific minor characteristics subgroups. HCTE model is designed to be hierarchical so that the specification of cloud products can be presented in a layered model in which there is no limit on dimensions and the number of layers. Also, the dimensions of layers are designed to be independent from each other. So, each major characteristics group can has its own specific number of layers. This characteristic of HCTE makes it extendable so that enables us to update our plan for any new upcoming version of products with new specifications or features. The cloud characteristics introduced in previous section are categorized and presented using HCTE model in Figure. 1.

\footnotetext{
${ }^{1}$ Hierarchical Cloud Taxonomy Engine
} 


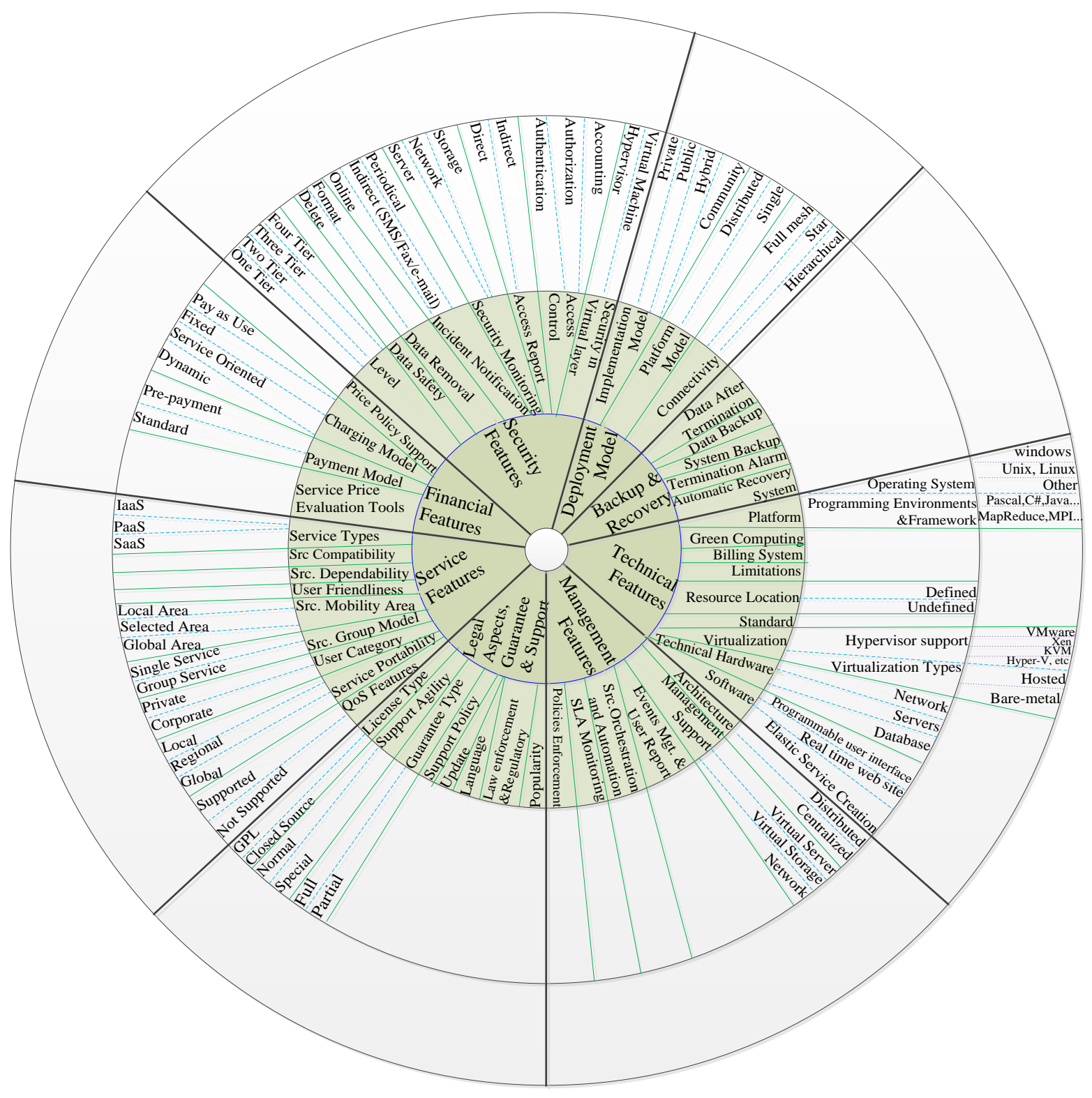

Figure 1. Taxonomy Design

\section{Elements of HCTE Taxonomy Model}

Author names and affiliations are to be centered beneath the title and printed in Times New Roman 12-point, non-boldface type. (See example below)

There are varieties of software products which can be used to establish a cloud platform. In this situation, cloud developers need some software tools to compare different software and choose the best matching product. To reach this goal, HCTE model was introduced in the previous section that provides comparison tools to make decisions based on characteristics of the products. In this section we introduce the elements of HCTE model described in previous section. HCTE is a new method for distinguishing characteristics of cloud products which classify them in eight different major groups. These major groups are (1) deployment model, (2) technical features, (3) security features, (4) service features, (5) management features, (6) financial features, (7) backup and recovery model, and finally (8) legal aspects as well as guarantee \& Support. The current findings in this section add to a growing body of literature on possible features of cloud 
computing products. In continue these categories as well as their components are described.

\subsection{Deployment Model}

4.1.1 Implementation Model: Cloud computing can be implemented in private, public, community and hybrid models. Each model has its own characteristics and design configuration. Some measureable characteristics are: cloud ownership, cloud manager, data center location, expandability, security, charging model, data hosting, efficiency and, cloud access pattern.

4.1.2 Platform Model: Supporting data centers constitute the model of cloud platform, which are single or distributed. In centralize data centers, which are platform of a cloud, platform is located in one geographical location, while in enterprise model, data centers are scattered along different locations.

4.1.3 Connectivity: Connectivity in cloud refers to the connection topology of data centers to form a cloud platform. The most important methods are full mesh, star and hierarchical models. In full mesh, there is a thorough peer-to-peer connection between all of the data centers. In the star model the architecture of the cloud looks like a star in which some data centers are located at the center of connection. In the hierarchical model, the data centers are handled in a hierarchical tree architecture in which there are different levels with different priority, management and access pattern.

\subsection{Technical Features}

4.2.1 Platform: Cloud Platform requires acquiring an operating system, programming environments and also supported applications and frameworks. There are various operating systems which cloud products may support, some of them are: windows, Linux, Solaris and so on. Programming environments and Frameworks is the other considerable issue in this section in which, the programming languages and runtime environments are mandatory. Moreover, considering supported application software, APIs, special domain, script and wizard are important items.

4.2.2 Virtualization: Virtualization technology acts as the base of cloud computing and provide helpful capabilities like resource abstraction and virtual machine migration. This technology is available by taking advantage of a hypervisor. So, cloud solution support from different powerful hypervisors and also the virtualization types are important factors to select a cloud product. Cloud products may support different hypervisors such as VMware, Xen, KVM and etc, as well as a hosted and bare-metal virtualization types.

4.2.3 Software: Important characteristics which are expected from cloud software are supporting a programmable user interface, real time web site and elastic service creation. Programmable user interface includes cloud software support from programmable control panels, attributes, environments, and programming languages. Real time web sites take advantage of technologies which enable users to receive information as it publishes by the authors. Elastic service creation refers to elastic cloud software support as well as simplicity of application creation.

4.2.4 Hardware Support: Cloud products may be compatible with only some specific hardware. So, network, server and database compatibility of cloud products should be explored before using them. In network facilities, the connection types between cloud modules should be considered which may be established using either cable or wireless 
media. In servers, characteristics of processing resources and server models should be considered. In database, the technology used to store data at local and a remote location should be considered.

4.2.5 Resource Location: This feature shows whether the cloud users are permitted to choose the location of cloud resources in advance of service delivery. Moreover, it defines whether the locations of resources are reported to cloud users during service delivery. We categorize locations of resources into defined and undefined locations. In the former type, resource locations are known while in the latter it is unknown places.

4.2.6 Standard: To make different cloud products interoperable and compatible with each other, standardization is a vital solution in all fields of technology. Standardization should be considered in different fields of cloud computing including cloud architectures, protocols, service identifiers, description languages, formats, virtualization, and servicelevel agreements. There are several organizations attempting to create such standards, including IBM, Sun Microsystems, DTMF, Intel, and Cisco.

4.2.7 Billing System: Billing system consisted of tools, institutions, instruments governed by laws, rules, procedures and standards to calculate and transfer the monetary value between parties discharging mutual obligations. Cloud works on a pay per use and charges users on the resources that are used out of the total resources that may be allocated to a particular. This feature shows the presence of billing system in the cloud product.

4.2.8 Limitations (H/S): Cloud products may have various technical hardware or software limitations, such as limitations in virtual part, cloud software and network limitations. Virtual layer limitations include maximum number of supported virtual machines, live virtual machines, virtual hosts and virtual data centers. Cloud environments limitations include maximum number of call APIs, users, site views, site creates and pages. Moreover, cloud products may have limitations in compatibility with other products. Network limitations include maximum bandwidth and storage allowed for each user.

4.2.9 Green Computing support: Green computing is a long-lasting concept which has become important in cloud computing in last few years. The goal of green computing is to reduce the huge energy consumption of modern cloud datacenters and maximize their energy efficiency during service life time. This feature shows whether there is such capability in the cloud products.

\subsection{Security Features}

4.3.1 Level: Based on security grade, security of data centers are divided into four tiers from one to four. Each tear has its own specific requirements. Simplest level of security is tier one and the highest level is tier four with fully redundant and strong access controls.

4.3.2 Data Safety: Data safety is the level of trust created for users that the data is safe from any hazardous defects. Data safety can be reached through encryption, locality and isolation of data. Encryption is done using data encoding so that only authorized party has access to it. Data isolation protects users from eavesdropping of other users or any malfunction created by hackers. 
4.3.3 Data Removal: Data removal is the process of eliminating unwanted data. Data Removal could be done in two ways, deleting the data and formatting the location. In case of deletion, data is removed; but, still exist. In the case of formating, data is unrecoverable.

4.3.4 Incident Notification : Incident notification or security reporting is one of the special features in cloud computing which can be online, indirect by SMS, Fax, e-mail or periodical probing. Incident notification includes the detected event, name, and time of occurrence.

4.3.5 Security Monitoring: Security monitoring is an appliance-based solution which provides control over the cloud by establishing a monitoring system. Security monitoring is done for servers, network and storage devices. It provides security events and security threats by collecting information from security devices and creates compliance reporting.

4.3.6 Security in Virtual layer: Security in virtual layer includes security of virtual machines and hypervisors. A virtual machine in cloud may be damaged or destroyed. The main threat that virtual machines are confronted with is their movement to other hardware systems and their probable combinations with other virtual machines. Hypervisor layer should be secure to ensure reliable and secure access control of virtual resources.

4.3.7 Access Control: Access control is a selective method to maintain confidentiality, integrity and availability in cloud services. Access mechanism includes authentication, authorization and accounting (AAA). It refers to security architecture for distributed systems, which controls users' access to cloud services, manage their access level, and also $\log$ the amount of resources they have used.

4.3.8 Access Report: Most of the cloud product has access report capability that provides reports for users and providers. Accessing to the reports can be provided based on direct and indirect methods.

\subsection{Service Features}

4.4.1 Service Types: Three main types of cloud computing services are Infrastructure as a service (IaaS), platform as a service (PaaS) and software as a service (SaaS). Infrastructure services deliver physical resources such as CPU, storage and network bandwidth. Platform services are those like programming, test and development tools and environments that help developers to accelerate the process of software development. Software services are those like Oracle $\mathrm{CRM}^{2}$ and Google Docs, in which specific software is delivered as a whole solution to customers.

4.4.2 Service Mobility Area: Service mobility area is the coverage area that indicates locations where the users can move on and still get their desired services. Mobility areas are divided into three categories which are local, global and selected area. In local mobility, cloud services are accessible only in a specific location. On the other hand, in global mobility services, users can move along all the covered area and access to their desired services. In selected area mobility, customers can access to their desired services from predefined locations.

4.4.3 Service Compatibility: Compatibility of services ensures operability of cloud services with other cloud software and tools. This characteristic comes from level of compliance with standards.

\footnotetext{
${ }^{2}$ Customer Relationship Management
} 
4.4.4 Service Dependability: Service dependability denotes whether the deliverable cloud services are dependable to a specific hardware, software, platform, etc. and what the level of dependability is.

4.4.5 User Friendliness: User friendliness shows how easy the user can handle the solution in an interactive model. The more user-friendly is a service, the more attractive is for users.

4.4.6 Service Group Model: Service group model indicates that whether the solution is intended to be used by single users or by a group of users. Also, if the cloud product is intended to be used by a group of users, it shows the maximum online users.

4.4.7 User Category: Some cloud products support a predefined user category that has different access level to various services. We can divide the user category into private and corporate. Corporate users often have higher access level than private users to different options.

4.4.8 Service Portability: Service portability shows the capability of the cloud product to transfer the cloud services between different regions under the coverage of the cloud infrastructure in face of sudden occurrence of undesirable situations like hazards.

4.4.9 QoS3 Features: Quality of service shows the grade of satisfaction that the users sense and can be realized from various parameters, like end to end delay, jitter in the network, the number of out of service occurrence and so on. These parameters are often agreed between costumers and the cloud providers based on their agreements. We consider the presence of a capability to change and monitor the QoS parameter in the middle of service operation as an important factor for service quality.

\subsection{Management Features}

4.5.1 Architecture: Management model is one of the most important features of cloud products which can be implemented in centralized or distributed structure. If the cloud product has centralized management architecture, the whole cloud is managed from a central point. On the other hand, in distributed management architecture there are many management entities distributed in different locations of the cloud that communicate with each other based on a specific connection topology.

4.5.2 Management Support: One of the functionalities expected from a cloud product to support is managing various domains such as resource management and service management. We divide resource management into virtual server, virtual storage and network domains separately.

4.5.3 Policies Enforcement: Policy enforcement capability is capability to apply policies involved in cloud service delivery related to provider, cloud auditor, cloud broker and cloud carrier. Besides, governments should have a capability to enforce their desired policies to the domain of cloud computing.

4.5.4 SLA Monitoring: This feature shows whether cloud products generate reports so that users can monitor their resource consumption and also their instantaneous charge.

${ }^{3}$ Quality of Service 
Moreover, this feature depicts whether the users can monitor fulfillment of the terms and conditions in SLA.

4.5.5 Service Orchestration and Automation: Service orchestration and automation is the automated arrangement, coordination, and management of cloud services. This feature depicts the capability of cloud products to automatically manage the process of service provisioning for users.

4.5.6 Events Management \& User Report: This feature describes whether the cloud product informs the users about the occurrence of internal events and whether it provides a report to the cloud users. Also, it shows whether the cloud users can manage their services according to their preferences.

\subsection{Financial Features}

4.6.1 Charging Model: Charging service model defines the cost associated with service catalog selected by cloud users. Different cloud providers use various charging models such as pay as use, fixed, service oriented and dynamic. In Pay as use model, users pay only for the period of time and the amount of resources they use. In the fixed model, a fixed charge is considered for each service. In service oriented model, charging computation depends on the service characteristics. In dynamic model, charging is dynamic and may depend to various factors such as auction, sale and so on.

4.6.2 Price Policy Support: This feature states whether the cloud product supports price policy mechanism.

4.6.3 Payment Model: Users of the cloud services can pay their charges through various models, such as cache or by check, offline or online, and bundled or partial.

4.6.4 Service Price Evaluation Tools : This feature shows the presence of service evaluation tools in cloud products. This tool is an important aid for users to compute their costs and to compare different scenarios of service consumption so that they can choose the best options.

\subsection{Backup \& Recovery Model}

4.7.1 Data Backup: Data backup is the process of copying and archiving data in a safe repository that helps to retrieve data in face of a plausible disaster. This feature shows whether the cloud product has a data backup procedure.

4.7.2 System Backup: System backup refers to the copying and archiving of system configuration data so that it can be used to restore the system after data loss. We divide system backup scenarios into single and redundant models. In former method, system backup data are stored in a single location, while in latter case, system backup data are stored in various locations.

4.7.3 Data after Termination: This feature shows the way a cloud product treats with the users' data after they stop their service usage or after the defined allowable time limit of user service. It depicts whether the cloud product completely removes the data so that it becomes unrecoverable.

4.7.4 Termination Alarm: This feature shows whether the cloud product alarms the users about the termination of their services. 
4.7.5 Automatic Recovery System: This feature shows whether there is any automatic recovery system available in the cloud product. Presence of an automatic recovery system helps to easily resuscitate the cloud service after a catastrophic system crash.

\subsection{Legal Aspects, Guarantee \& Support}

4.8.1 License Type: License type defines the type of user access, use and publishing of cloud software. There are two main license types, GPL and closed source. In general public license, source code is available for developer; while in closed source, source code is not available.

4.8.2 Support Policy: Support policy shows how the cloud software provider supports its product. Class of support and level of service defines the support policy.

4.8.3 Support Agility: Support agility shows the reaction speed of cloud product provider to fix any problem such as its product malfunctioning. It has two category of normal and special. The faster the provider fixes the problem, the more attractive the service become.

4.8.4 Guarantee Type: Guarantee type shows the level of guarantee that the cloud provider provides for its products. Moreover, it describes the time period and guarantee details. More precisely, it defines if the cloud product has any defect, how the vendor will go about rectifying them.

4.8.5 Update: This feature shows whether the cloud product providers prepare their product updates available for release.

4.8.6 Language: This feature shows the number of languages supported by cloud software. Supporting form local language is an important concern of local users.

4.8.7 Law enforcement \& Regulatory Compliance: Each country has its own law and regulations. This feature shows whether the cloud product provides capability to define policies in such a way that local law and regulations become fulfilled.

4.8.8 Popularity: Popularity of products has direct relation to universal acceptance around the world. Generally, most of the popular cloud products have more functionality in comparison with other cloud products. Usually special products of famous brands become popular because of their specific advantages.

\section{Proposed Method for Product Evaluation}

In previous sections we introduced the HCTE model as well as its elements including thorough characteristics of cloud systems. HCTE considered eight different categories to cover main characteristics of cloud products. In this section, we propose a comparison process based on introduced characteristics and designed taxonomy. This comparison method is an effective method suitable for cloud providers and developers to select the best matching products in accordance with their preferences and policies.

In this method, score of each cloud product is calculated based on both product characteristics and user requirements. Then, different products are graded and ranked based on calculated score. In the next step, the matching percentage of each cloud product to user requirements is calculated. Finally, the best product is suggested to the user based on computed score and matching percentage which has emphasis on compatibility ratio of 
product to user's demands and priorities. The score given to a cloud product is computed based on the following equations.

$$
\begin{aligned}
& \text { Score }(\text { product })=\sum_{i=1}^{8} X_{i} \times \text { ScFeature }_{i} \\
& \text { ScFeature }_{i}=\sum_{j} X_{j} \times \text { ScSubFeature } \\
& \text { ScSubFeature }=\left\{\begin{array}{lr}
+1 \text { or } 0 & Y / N \text { feature } \\
\frac{\# \text { Positive Features }}{\text { Total } \# \text { of Features }} & \text { Numbered features }
\end{array}\right. \\
& X_{i}=\left\{\begin{array}{lll}
1 & \text { if } \text { characteri stic } \text { }_{\mathrm{i}} \text { is stated by theuser } \\
0 & \text { otherwise }
\end{array}\right.
\end{aligned}
$$

Equation 1 is calculated based on main eight features presented in section 4. In this equation, variable ${ }^{X_{i}}$ refers to expected characteristics of cloud products. If a feature is stated by a user as a preference, ${ }^{X_{i}}$ is set to be one; otherwise it is set to be zero. All features have their dedicated sub-features which are shown in the second level of the taxonomy graph. In equation 2, score of each feature is calculated through summation of its sub-features scores. All characteristics introduced in previous sections for cloud computing products can be categorized as deterministic or linguistic variables. While variables in mathematics usually take numerical values for deterministic type, the nonnumeric linguistic variables are often used to facilitate the expression of rules and facts [18]. A linguistic variable such as age may have a value such as young or its antonym old. However, the great utility of linguistic variables is that they can be modified via linguistic hedges applied to primary terms[18].

Table 1 shows the types and value range for features and sub-features introduced in HCTE. Moreover, in this table the range of variables are depicted in front of them as well as their variable types. More precisely, a numerical min-max range for deterministic features and a linguistic min-max range for linguistic features are defined. We divide deterministic variable types into two categories which are $\mathrm{Y} / \mathrm{N}$ (yes or no) or the value numbering features. In the former category, the deterministic values of one and zero are considered for Yes and No types respectively such that score one is added to the subfeature grade if the cloud product has the intended characteristics, and zero otherwise. In latter category, the number of supported target characteristics are counted and considered as their value. Furthermore, the value of linguistic variables is considered in the range between "Very Low" and "Very High". In order to define numerical values for linguistic variables so that they can be entered in our numerical computations, the translation map shown in table 2 is used to convert linguistic variables to deterministic values.

Table 1. Feature Types and their Value Range

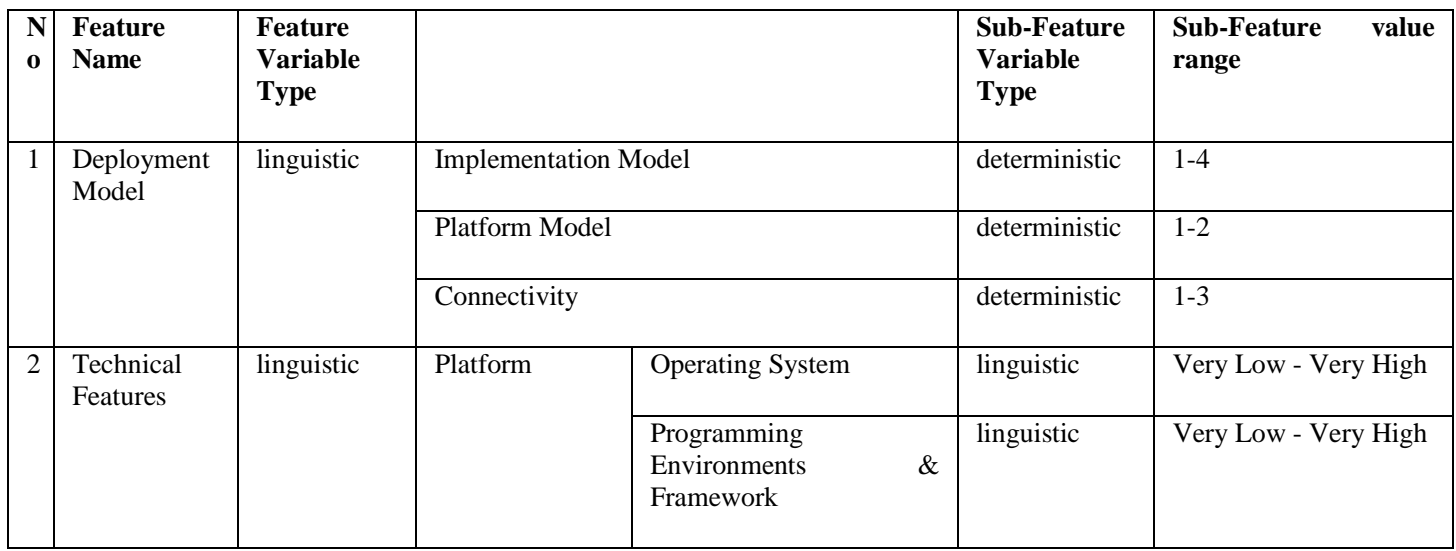




\begin{tabular}{|c|c|c|c|c|c|c|c|}
\hline & & & \multirow[t]{2}{*}{ Virtualization } & \multicolumn{2}{|c|}{ Virtualization Type } & deterministic & $1-2$ \\
\hline & & & & \multicolumn{2}{|c|}{ Hypervisor support } & deterministic & $1-5$ \\
\hline & & & \multirow[t]{3}{*}{ Software } & \multicolumn{2}{|c|}{$\begin{array}{ll}\text { Programmable user } \\
\text { interface }\end{array}$} & deterministic & $\mathrm{Y} / \mathrm{N}$ \\
\hline & & & & \multicolumn{2}{|c|}{ Real time web site } & deterministic & $\mathrm{Y} / \mathrm{N}$ \\
\hline & & & & \multicolumn{2}{|c|}{ Elastic Service Creation } & deterministic & $\mathrm{Y} / \mathrm{N}$ \\
\hline & & & Technical & \multicolumn{2}{|c|}{ Server } & deterministic & $\mathrm{Y} / \mathrm{N}$ \\
\hline & & & Support & \multicolumn{2}{|c|}{ Network } & deterministic & $\mathrm{Y} / \mathrm{N}$ \\
\hline & & & & \multicolumn{2}{|c|}{ Database } & deterministic & $\mathrm{Y} / \mathrm{N}$ \\
\hline & & & \multicolumn{3}{|c|}{ Resource Location } & deterministic & $\begin{array}{l}\text { Y(Defined)/N(Undefin } \\
\text { ed) }\end{array}$ \\
\hline & & & \multicolumn{3}{|l|}{ Standard } & deterministic & $\mathrm{Y} / \mathrm{N}$ \\
\hline & & & \multicolumn{3}{|l|}{ Billing System } & deterministic & $\mathrm{Y} / \mathrm{N}$ \\
\hline & & & \multicolumn{3}{|l|}{ Limitations } & linguistic & Very Low- Very High \\
\hline & & & \multicolumn{3}{|c|}{ Green Computing Support } & deterministic & $\mathrm{Y} / \mathrm{N}$ \\
\hline \multirow[t]{2}{*}{3} & \multirow{13}{*}{$\begin{array}{l}\text { Security } \\
\text { Features }\end{array}$} & \multirow[t]{13}{*}{ linguistic } & \multicolumn{3}{|l|}{ Level } & deterministic & $1-4$ \\
\hline & & & \multicolumn{3}{|l|}{ Data Safety } & linguistic & Very Low- Very High \\
\hline & & & \multicolumn{3}{|l|}{ Data Removal } & deterministic & $\mathrm{Y}$ (Format)/N (Delete) \\
\hline & & & \multicolumn{3}{|c|}{ Incident Notification } & linguistic & Very Low- Very High \\
\hline & & & \multirow{3}{*}{\multicolumn{2}{|c|}{ Security Monitoring }} & Server & deterministic & $\mathrm{Y} / \mathrm{N}$ \\
\hline & & & & & Network & deterministic & $\mathrm{Y} / \mathrm{N}$ \\
\hline & & & & & Storage & deterministic & $\mathrm{Y} / \mathrm{N}$ \\
\hline & & & \multirow{2}{*}{\multicolumn{2}{|c|}{$\begin{array}{l}\text { Security in Virtual } \\
\text { layer }\end{array}$}} & Virtual Machine & deterministic & $\mathrm{Y} / \mathrm{N}$ \\
\hline & & & & & Hypervisor & deterministic & $\mathrm{Y} / \mathrm{N}$ \\
\hline & & & \multirow{3}{*}{\multicolumn{2}{|c|}{ Access Control }} & Authentication & deterministic & $\mathrm{Y} / \mathrm{N}$ \\
\hline & & & & & Authorization & deterministic & $\mathrm{Y} / \mathrm{N}$ \\
\hline & & & & & Accounting & deterministic & $\mathrm{Y} / \mathrm{N}$ \\
\hline & & & \multicolumn{3}{|l|}{ Access Report } & deterministic & $\mathrm{Y}($ Direct $) / \mathrm{N}($ Indirect $)$ \\
\hline 4 & \multirow{6}{*}{$\begin{array}{l}\text { Service } \\
\text { Features }\end{array}$} & \multirow[t]{6}{*}{ linguistic } & Service Types & & & deterministic & $1-3$ \\
\hline & & & Service Mobili & Area & & linguistic & $\begin{array}{l}\text { Very Low(Local)- } \\
\text { Very High(Global) }\end{array}$ \\
\hline & & & Service Compa & bility & & deterministic & $\mathrm{Y} / \mathrm{N}$ \\
\hline & & & Service Depen & bility & & deterministic & $\mathrm{Y} / \mathrm{N}$ \\
\hline & & & User Friendlin & & & deterministic & $\mathrm{Y} / \mathrm{N}$ \\
\hline & & & Service Group & Iodel & & deterministic & $\mathrm{Y}$ (Group)/N(Single) \\
\hline
\end{tabular}




\begin{tabular}{|c|c|c|c|c|c|c|}
\hline & & & \multicolumn{2}{|c|}{ User Category } & deterministic & $1-2$ \\
\hline & & & \multicolumn{2}{|c|}{ Service Portability } & linguistic & $\begin{array}{l}\text { Low(Local)- } \\
\text { Medium(Regional)- } \\
\text { High(Global) }\end{array}$ \\
\hline & & & \multicolumn{2}{|l|}{ QoS Features } & deterministic & $\begin{array}{l}\text { Y(Supported)/N(Not } \\
\text { Supported) }\end{array}$ \\
\hline \multirow[t]{8}{*}{5} & \multirow{8}{*}{$\begin{array}{l}\text { Management } \\
\text { Features }\end{array}$} & \multirow[t]{8}{*}{ linguistic } & \multicolumn{2}{|l|}{ Architecture } & deterministic & $1-2$ \\
\hline & & & \multirow[t]{3}{*}{$\begin{array}{l}\text { Management } \\
\text { Support }\end{array}$} & Virtual Server & deterministic & $\mathrm{Y} / \mathrm{N}$ \\
\hline & & & & Virtual Storage & deterministic & $\mathrm{Y} / \mathrm{N}$ \\
\hline & & & & Network & deterministic & $\mathrm{Y} / \mathrm{N}$ \\
\hline & & & \multicolumn{2}{|c|}{ Policies Enforcement } & deterministic & $\mathrm{Y} / \mathrm{N}$ \\
\hline & & & \multicolumn{2}{|c|}{ SLA Monitoring } & deterministic & $\mathrm{Y} / \mathrm{N}$ \\
\hline & & & \multicolumn{2}{|c|}{ Service Orchestration and Automation } & deterministic & $\mathrm{Y} / \mathrm{N}$ \\
\hline & & & \multicolumn{2}{|c|}{ Events Management \& User Report } & deterministic & $\mathrm{Y} / \mathrm{N}$ \\
\hline \multirow[t]{4}{*}{6} & \multirow{4}{*}{$\begin{array}{l}\text { Financial } \\
\text { Features }\end{array}$} & \multirow[t]{4}{*}{ linguistic } & \multicolumn{2}{|c|}{ Charging Model } & deterministic & $1-4$ \\
\hline & & & \multicolumn{2}{|c|}{ Price Policy Support } & deterministic & $\mathrm{Y} / \mathrm{N}$ \\
\hline & & & \multicolumn{2}{|c|}{ Payment Model } & deterministic & $1-2$ \\
\hline & & & \multicolumn{2}{|c|}{ Service Price Evaluation Tools } & deterministic & $\mathrm{Y} / \mathrm{N}$ \\
\hline \multirow[t]{5}{*}{7} & \multirow[t]{5}{*}{$\begin{array}{l}\text { Backup \& } \\
\text { Recovery }\end{array}$} & \multirow[t]{5}{*}{ linguistic } & \multicolumn{2}{|l|}{ Data Backup } & deterministic & $\mathrm{Y} / \mathrm{N}$ \\
\hline & & & \multicolumn{2}{|c|}{ System Backup } & deterministic & $\mathrm{Y} / \mathrm{N}$ \\
\hline & & & \multicolumn{2}{|c|}{ Data After Termination } & deterministic & $\mathrm{Y} / \mathrm{N}$ \\
\hline & & & \multicolumn{2}{|c|}{ Termination Alarm } & deterministic & $\mathrm{Y} / \mathrm{N}$ \\
\hline & & & \multicolumn{2}{|c|}{ Automatic Recovery System } & deterministic & $\mathrm{Y} / \mathrm{N}$ \\
\hline \multirow[t]{8}{*}{8} & \multirow{8}{*}{$\begin{array}{l}\text { Legal } \\
\text { Aspects, } \\
\text { Guarantee \& } \\
\text { Support }\end{array}$} & \multirow[t]{8}{*}{ linguistic } & \multicolumn{2}{|l|}{ License Type } & deterministic & $\mathrm{Y}(\mathrm{GPL}) / \mathrm{N}(\mathrm{Closed})$ \\
\hline & & & \multicolumn{2}{|c|}{ Support Policy } & linguistic & Very Low- Very High \\
\hline & & & Support Agili & & linguistic & $\begin{array}{l}\text { Very Low(Normal)- } \\
\text { Very High(Special) }\end{array}$ \\
\hline & & & Guarantee Ty & & deterministic & Y(Full)/N(Partial) \\
\hline & & & Update & & deterministic & $\mathrm{Y} / \mathrm{N}$ \\
\hline & & & Language & & linguistic & Very Low- Very High \\
\hline & & & Law enforcen & gulatory Compliance & deterministic & $\mathrm{Y} / \mathrm{N}$ \\
\hline & & & Popularity & & linguistic & Very Low- Very High \\
\hline
\end{tabular}


Table 2. Translation Map from Linguistic to Deterministic Variables

\begin{tabular}{|l|l|}
\hline Linguistic Value & Deterministic Value \\
\hline Very Low & 1 \\
\hline Low & 2 \\
\hline Medium & 3 \\
\hline High & 4 \\
\hline Very High & 5 \\
\hline
\end{tabular}

\section{Experimental and Evaluation Results}

In this section, we present a practical cloud product comparison procedure to evaluate the capability of our proposed HCTE representation model as well as our proposed comparison process. To avoid facing special rights related to cloud vendors, we consider three hypothetical cloud products without any brand name. So, as a case study, cloud products 1,2, and 3 are considered as well as a customer who needs a cloud product with features depicted in Figure. 5 using HCTE model. Besides, we assume that aforementioned cloud products have the features shown in Figure. 2, 3, and 4 using HCTE model. At the first stage, we compute the score of each product regardless of the customer's requirements by considering value 1 for all Xi parameters defined in equations 1 and 2, the results of which is depicted in Figure. 6. This score shows the closeness of assumed product to the ideal case that has all the defined features presented using HCTE in Figure. 1. At the second stage, we compute the score of cloud products based on the equations defined in section 5 as well as the matching percentage between the cloud products and the user requirements, the result of which is depicted in Figure. 7. The matching percentage shows the percentage of features required by the customer which are present in the cloud product. It can be deduced from the matching percentage and computed score obtained from these calculations that product number one has the highest match to the cloud user requirements. However, it is important to note that if the user has a more limited choice that can only select from product 2 and 3, the cloud product selection process becomes more delicate. More precisely, although the score of product 2 is higher than product 3 regardless of the user's requirements, but the score and matching percentage of product 3 is higher than product 2 considering the user's requirements. So, our proposed system suggests cloud product 3 to user due to its higher compatibility to user's requirements. 


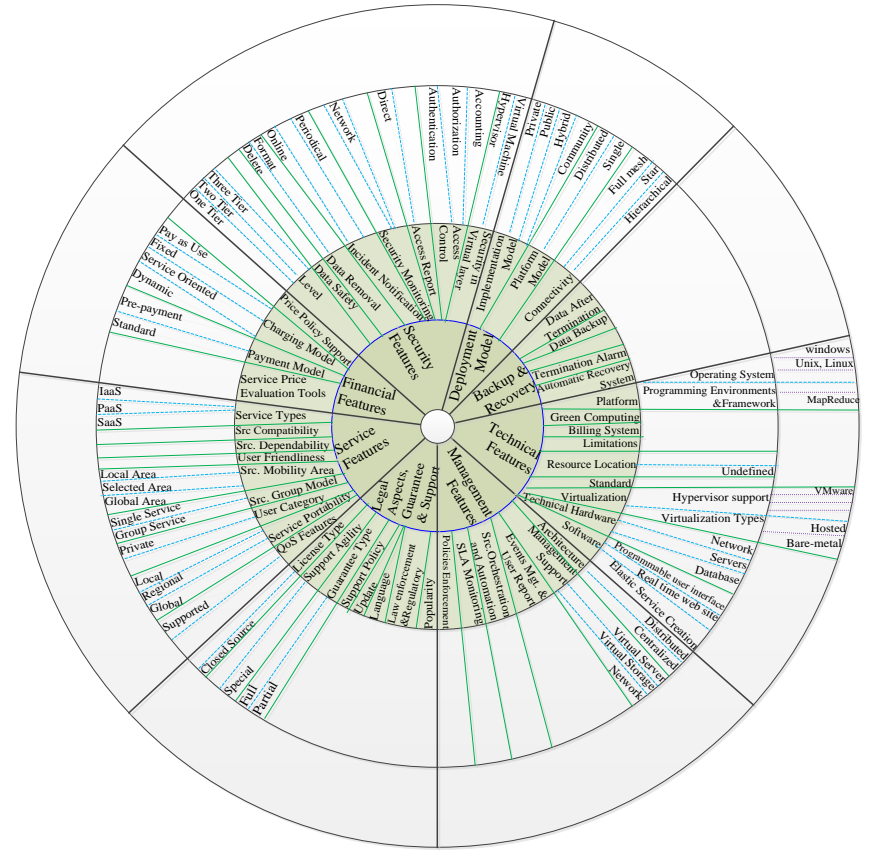

Figure 2.Product1 Taxonomy

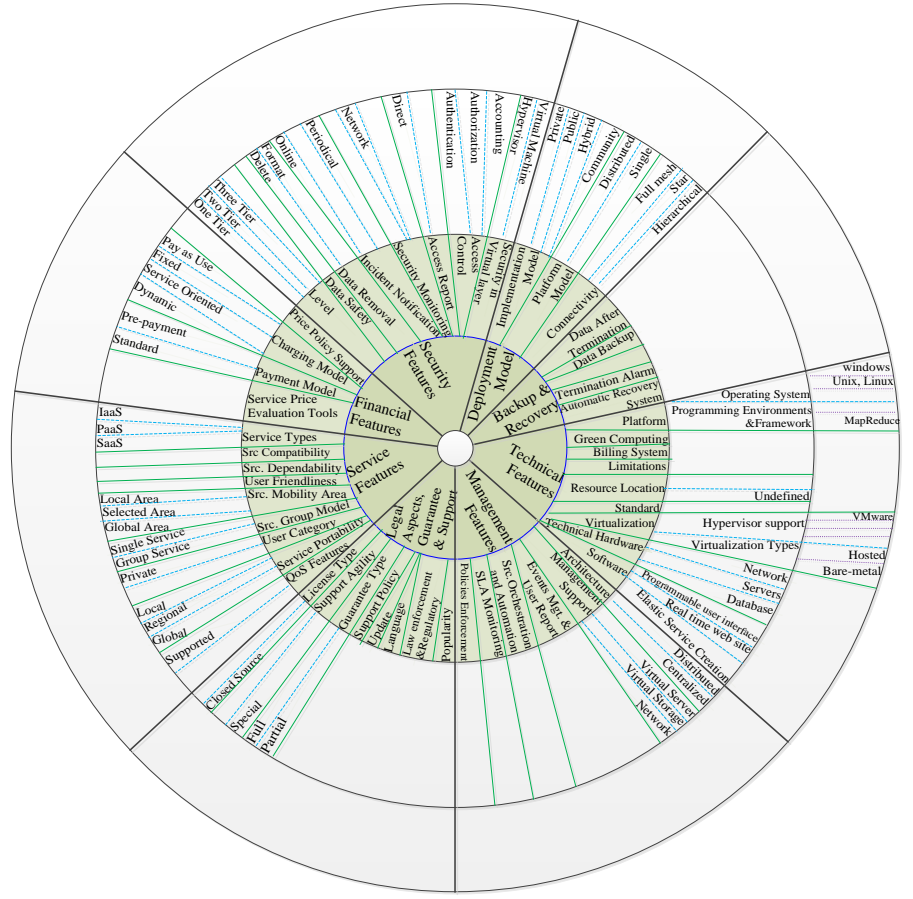

Figure 4. Product3 Taxonomy

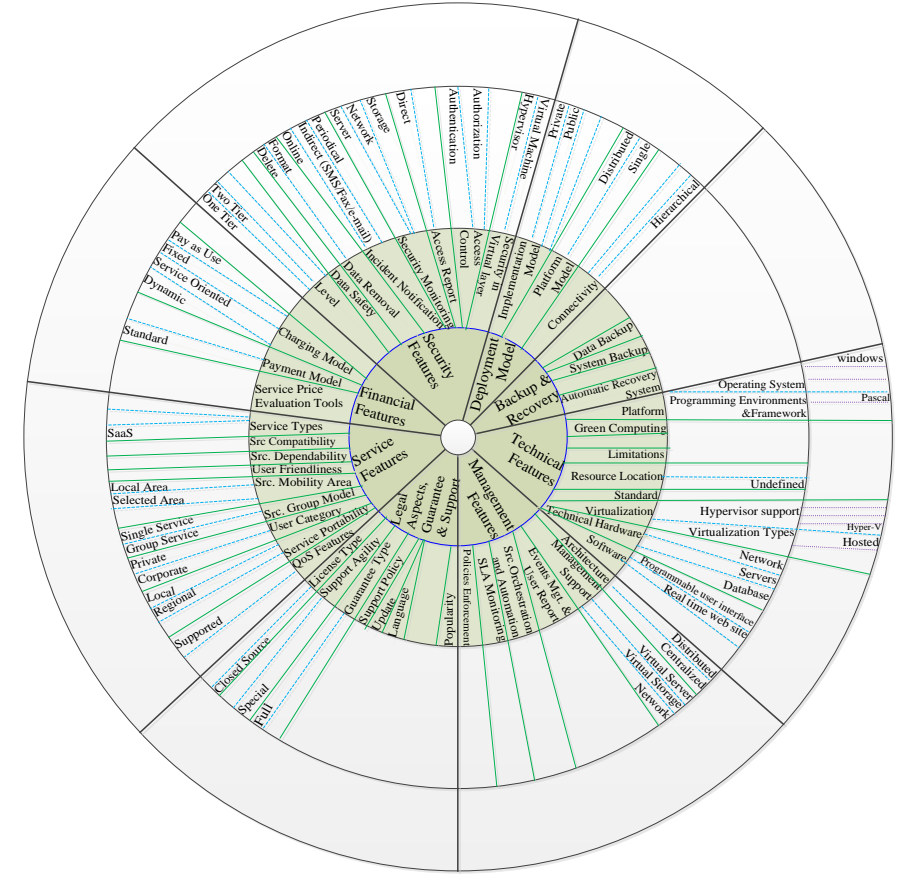

Figure 3. Product2 Taxonomy

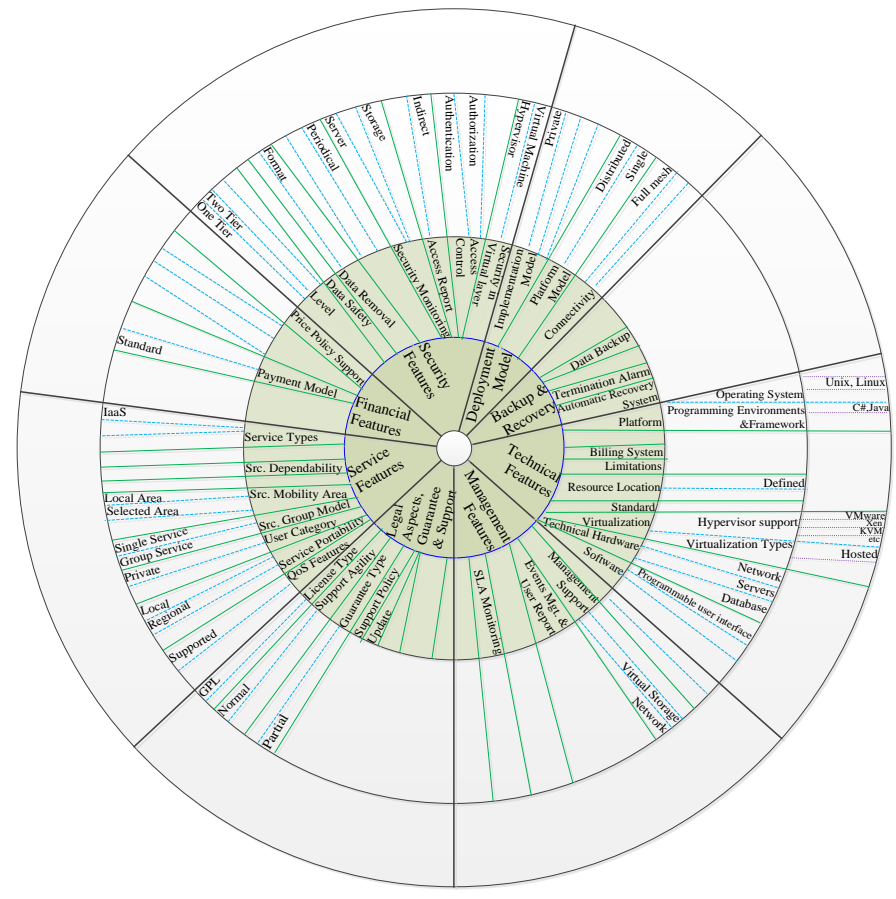

Figure 5. Requirements of Cloud User 


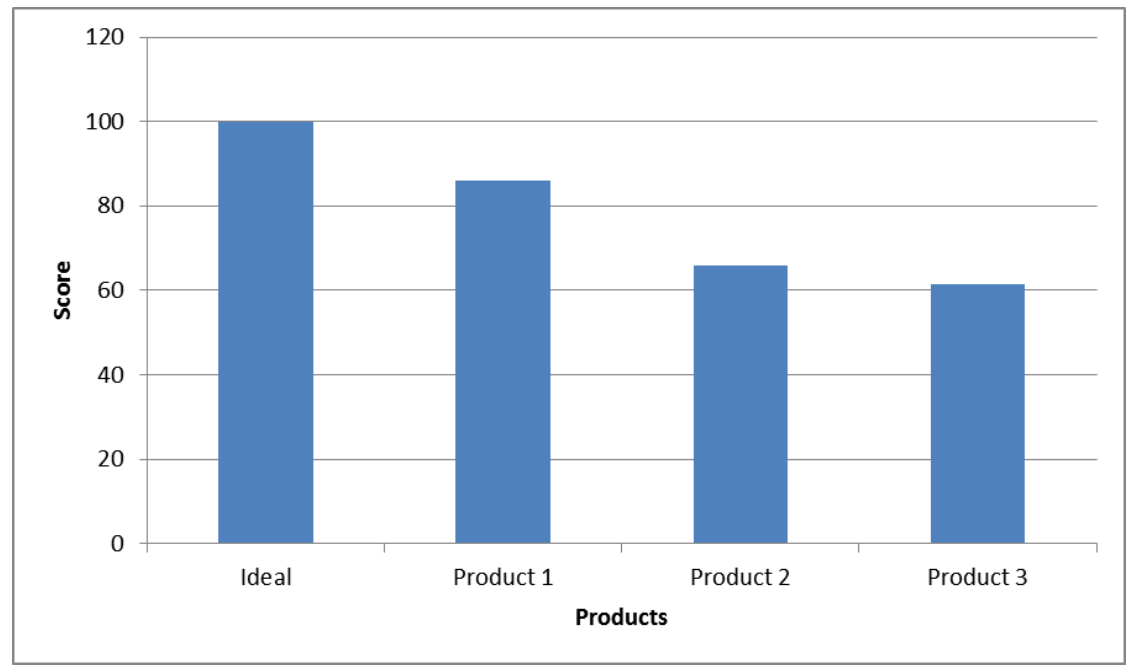

Figure 6. Cloud Products Score Regardless of the Customer's Requirements

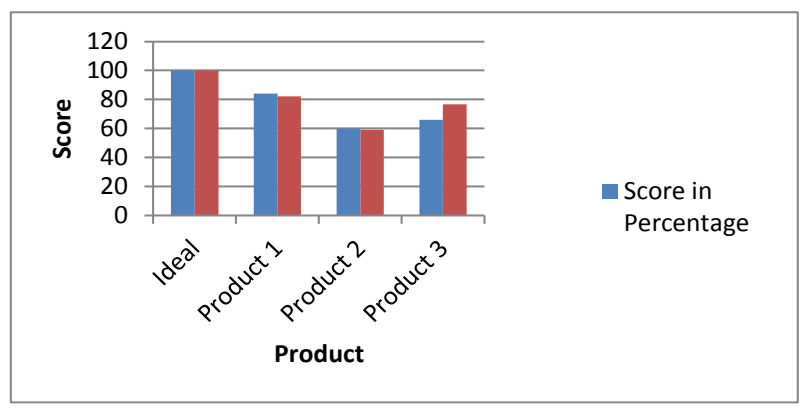

\section{Figure 7. Comparison of Cloud Products based on the Customer's Requirements}

\section{Conclusion and Future Directions}

The purpose of this research study was to design and evaluate a smart cloud product evaluation system which offers the best cloud product to cloud customers or service provider. We have proposed HCTE as a representation model for cloud products features classification which facilitates the process of cloud product evaluation and selection. HCTE has investigated the mandatory features for different customers and compared them with specification of different cloud products in order to represent and compare cloud products. In HCTE, we have categorized all the possible features for cloud products in eight major categories including deployment model, technical features, security features, service features, management features, financial features, backup and recovery model, and finally legal aspects as well as guarantee \& Support. Besides, we have developed a cloud product evaluation system to compare and select appropriate cloud product based on the requirements stated by the customer. The proposed cloud product evaluation system calculates the matching percentage of the cloud products with user requirements as well as a score derived from the proposed equations in order to suggest the best product which has the highest match to user requirements. The empirical cloud features presented in this study provide a new understanding of all aspects of 
characteristics related to cloud computing that are very important for selecting a suitable product. It is important to note that the HCTE model and the evaluation methods which are used to represent and compare cloud products, can be applied to other sections of cloud computing such as cloud computing business models. Our future direction is aimed to devise a suitable cloud computing business model based on HCTE model. Another research direction is implementation of the proposed methods using real cloud management products.

\section{References}

[1] F. Hu, M. Qiu, J. Li, T. Grant, D. Taylor, S. McCaleb, et al., "A review on cloud computing: Design challenges in architecture and security," CIT. Journal of Computing and Information Technology, vol. 19, pp. 25-55, 2011.

[2] M. Hajibaba and S. Gorgin, "A Review on Modern Distributed Computing Paradigms: Cloud Computing, Jungle Computing and Fog Computing," CIT. Journal of Computing and Information Technology, vol. 22, pp. 69-84, 2014.

[3] M. Hamdaqa and L. Tahvildari, "Cloud Computing Uncovered: A Research Landscape," Advances in Computers, vol. 86, pp. 41-85, 2012.

[4] R. Buyya, C. S. Yeo, S. Venugopal, J. Broberg, and I. Brandic, "Cloud computing and emerging IT platforms: Vision, hype, and reality for delivering computing as the 5th utility," Future Generation computer systems, vol. 25, pp. 599-616, 2009.

[5] P. Mell and T. Grance, "The NIST definition of cloud computing (draft)," NIST special publication, vol. 800, p. 7, 2011.

[6] A. KS, T. A. Thomas, and G. Santhoshkumar, "International Journal of Advanced Research in Computer Science and Software Engineering," International Journal, vol. 3, 2013.

[7] M. S. Harnal and M. D. Bagga, "Cloud Computing: An Overview," International Journal, vol. 3, 2013.

[8] C. Höfer and G. Karagiannis, "Cloud computing services: taxonomy and comparison," Journal of Internet Services and Applications, vol. 2, pp. 81-94, 2011.

[9] D. R. Herrick, "Google this!: using Google apps for collaboration and productivity," in Proceedings of the 37th annual ACM SIGUCCS fall conference, 2009, pp. 55-64.

[10] L. Qian, Z. Luo, Y. Du, and L. Guo, "Cloud computing: an overview," in Cloud Computing, ed: Springer, 2009, pp. 626-631.

[11] F. Liu, J. Tong, J. Mao, R. Bohn, J. Messina, L. Badger, et al., "NIST cloud computing reference architecture," NIST special publication, vol. 500, p. 292, 2011.

[12] H. Li, C. Spence, R. Armstrong, R. Godfrey, R. Schneider, J. Smith, et al., "Intel cloud computing taxonomy and ecosystem analysis," IT-Intel Brief (Cloud Computing), 2010.

[13] C. Hoefer and G. Karagiannis, "Taxonomy of cloud computing services," in GLOBECOM Workshops (GC Wkshps), 2010 IEEE, 2010, pp. 1345-1350.

[14] L. Wang, G. Von Laszewski, A. Younge, X. He, M. Kunze, J. Tao, et al., "Cloud computing: a perspective study," New Generation Computing, vol. 28, pp. 137-146, 2010.

[15] T. Harris, "Cloud Computing Services-A Comparison," ed: From, 2010.

[16] B. P. Rimal, E. Choi, and I. Lumb, "A taxonomy and survey of cloud computing systems," in INC, IMS and IDC, 2009. NCM'09. Fifth International Joint Conference on, 2009, pp. 44-51.

[17] D. Hilley, "Cloud computing: A taxonomy of platform and infrastructure-level offerings," Georgia Institute of Technology, Tech. Rep. GIT-CERCS-09-13, 2009.

[18] L. A. Zadeh, Fuzzy sets, fuzzy logic, and fuzzy systems: selected papers vol. 6: World Scientific, 1996.

\section{Authors}

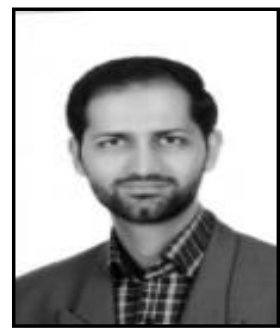

Ehsan Arianyan, He received the M.S. and PhD. degrees from Amirkabir University of Technology, Tehran, Iran, in 2010 and 2015, respectively. He is currently working as a researcher and project manager in Iran Telecommunication Research Institute (ITRC). He is the author of more than 10 peer-reviewed papers as well as 3 books related to cloud computing. His research interests include cloud computing, parallel computing, big data, and decision algorithms. 


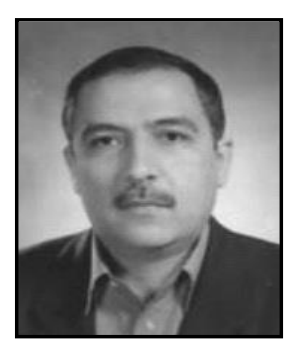

Mohammad Reza Ahmadi, He received the B.Sc. and M.Sc. degrees in Electrical Engineering and Communication Systems from K.N.T. University of Technology in 1986 and 1990 respectively. He received his Doctor degree in Communication Networks from Tokyo Institute of Technology, Tokyo, in 1997. Currently he is a project manager and researcher in Iran Telecommunication Research Center (ITRC). His research interests are network security focus on intrusion detection systems, Immune systems focus on network applications, data centers design \& implementation, cloud computing and big data.

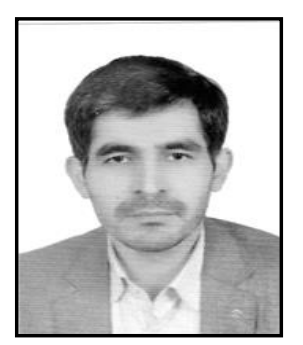

Davood Maleki, He received the B.E. degree from Amir Kabir Univ. of Technology in 1999 and M. E. degree in software engineering from Ferdowsi Univ. of Mashhad in 2004. His Thesis of M. E. was about network security using soft computing, evolutionary algorithms and immune systems. He works as a researcher assistant (from 2004) in the Dept. of Network Security in Iran Telecommunication Research Center (ITRC). His research interest includes distributed computing, security management of data center, cloud computing and big data. 